\title{
What evidence for the benefits of '5-a-day', a Mediterranean diet and sodium restriction on health?
}

Guidelines for healthcare professionals encourage the provision of dietary advice to promote healthy eating, especially to patients at risk of chronic disease. ${ }^{1}$ Yet the evidence base for dietary interventions relies heavily on epidemiological studies, which are subject to the challenges associated with observational research. Such problems include difficulties of assessing and measuring outcomes, misclassification, confounding and establishing causation. This reliance on epidemiological evidence may reflect the difficulty and cost of carrying out large-scale long-term randomised controlled studies of diet. ${ }^{2}$ In addition, there is a dearth of organisations willing to fund such research.

Recent publications have questioned the ' 5 -a-day' advice for fruit and vegetable consumption and population-level attempts to lower salt consumption. ${ }^{3,4}$ Studies of lifestyle advice are widely reported in the media, which may lead to public confusion about dietary advice when conclusions differ. Some researchers have urged a move from assessing how single foods or nutrients affect risk factors, to a consideration of the overall diet pattern, as this may overcome the risk of confounding the effect of one food type by others in the diet. ${ }^{5}$ The Mediterranean diet pattern is one of the most studied, since its identification in the late 1970s. Here, we provide an update of evidence for three aspects of dietary recommendations that feature regularly in the media-fruit and vegetable intake, salt reduction and the Mediterranean diet.

\section{Current advice}

UK dietary advice for adults includes recommendations to eat at least five portions of fruit and vegetables daily ( $400 \mathrm{~g}$ in total), reduce consumption of saturated fat, limit salt intake to no more than $6 \mathrm{~g}$ daily for adults, eat two portions of fish a week (one of which should be oily) and increase consumption of fibre-rich foods such as lentils, whole grains and nuts. ${ }^{1}$ This advice comes from public health and clinical guidance aimed at preventing obesity, diabetes and cardiovascular disease (CVD). However, most people in the UK don't meet the national guidelines. ${ }^{6}$ The most recent results of the National Diet Survey report that adults aged 19-64 years on average consumed 4.1 portions of fruit and vegetables per day, boys aged 11-18 years 3.0 portions per day and girls aged $11-18$ years 2.7 portions per day. ${ }^{6}$

Public health dietary advice from Europe, North America and Australia have similar recommendations. ${ }^{7-10}$ Some North American guidelines recommend the Mediterranean diet. ${ }^{11}$

\section{Fruit and vegetable consumption}

A Cochrane review and meta-analysis of randomised controlled trials investigating the effect of attempts to increase fruit and vegetable consumption on primary prevention of CVD found 10 trials with 1,730 participants.12 The studies included adult men and women at varying levels of CVD risk. The majority of the trials were conducted in developed

\footnotetext{
DTB CME/CPD

A CME/CPD module based on this article is available for completion online via BMJ Learning (learning.bmj.com) by subscribers to the online version of DTB. If prompted, subscribers must sign into DTB with their username and password. All users must also complete a one-time registration on BMJ Learning and subsequently log in (with a BMJ Learning username and password) on every visit. The answers to the multiple choice questions will be freely available on dtb.bmj.com on publication of the next issue of DTB.
}

countries. Four trials studied provision of advice and six studied provision of fruit and vegetables by the investigators. However, none of the trials reported clinical events as outcomes. Dietary advice compared with no dietary advice lowered systolic blood pressure by $3 \mathrm{mmHg}(95 \% \mathrm{Cl} 4.9$ to 1.1). No effect was found on total cholesterol and effects on diastolic blood pressure and low-density lipoprotein (LDL) cholesterol were not statistically significant. ${ }^{12}$ Trials of provision of fruit and vegetables were heterogeneous and short-term, and did not provide strong evidence suggesting a reduction in cardiovascular risk.

\section{Effect on health}

A systematic review of higher compared with lower consumption of fruit and vegetables in prospective cohort studies (20 studies including

760,629 participants from the USA, Europe and Asia) showed a 0.79 relative risk reduction for stroke ( $95 \% \mathrm{Cl} 0.75$ to 0.84$))^{13}$ The authors investigated the optimal number of daily portions of fruit and vegetables and suggested that there was a relative risk reduction for stroke of $32 \%$ for every $200 \mathrm{~g}$ incremental increase in fruit consumption, and a reduction of $11 \%$ for vegetables. ${ }^{13}$ While most of the studies controlled for known risk factors such as smoking, alcohol intake, blood pressure and physical activity, residual confounding may limit the reliability of the results. The effects of fruit and vegetable consumption on mortality were analysed in a systematic review of prospective cohort studies (16 studies from USA, Asia and Europe with 833,234 participants). ${ }^{3}$ The authors found that an increase of one additional serving per day resulted in a hazard ratio of $0.95(95 \% \mathrm{Cl} 0.92$ to 0.98$)$ for all-cause mortality and 0.96 for cardiovascular mortality ( $95 \%$ Cl 0.92 to 0.99 ). The hazard ratio for five servings a day, compared with none, was $0.74(95 \% \mathrm{Cl} 0.66$ to 0.82). There was no evidence of a further reduction in risk beyond five servings a day. ${ }^{3}$ However, only half of the studies controlled for physical activity.

Reviews of evidence for an effect on cancer and diabetes incidence have been less positive. In one review there was a non-significant hazard ratio for cancer mortality. ${ }^{3}$ Another found that high fruit and vegetable intake was inversely associated with CVD incidence, but that the results for cancer were unclear. ${ }^{14} \mathrm{~A}$ clinical review to update the World Cancer Research Fund's 
lifestyle recommendations found "no convincing evidence that fruits and vegetables play a role in cancer aetiology".15 Three systematic reviews examining diabetes incidence had inconsistent results, with small effects of borderline or no statistical significance. $14,16,17$

Only the systematic review of randomised controlled trials considered adverse events, reporting that advice to increase fruit and vegetable consumption increased flatulence and bowel movements. ${ }^{12}$

An Australian review that compared cost-effectiveness by disabilityadjusted life years for 23 interventions aimed at promoting fruit and vegetable intake found that an intervention run at community level was most cost-effective, although the researchers estimated it could only avert $5 \%$ of the disease burden attributed to low fruit and vegetable intake. ${ }^{18}$ Interventions that used individual dietary counselling, telephone contact and workplace promotion were not cost effective. A more recent UK-based health economic analysis used a Markov simulation model to estimate the cost-effectiveness of providing brief annual dietary intervention in primary care to a general population of healthy adults, free from chronic disease. ${ }^{19}$ The model assumed this would increase consumption by 0.5 portions a day, but found the strategy was 'unlikely to be cost-effective'.

\section{Mediterranean diet}

A major problem is inconsistency of definition of a Mediterranean-style diet. Components frequently cited in studies as markers for the diet include high consumption of olive oil in preference to saturated fat, legumes, whole-grain cereal, fruit and vegetables, and moderate consumption of wine. Some studies also include the moderate consumption of fish and dairy products and low consumption of poultry, meat, processed food, refined grains and sugar. ${ }^{20}$ Studies score adherence to a Mediterranean-style dietary pattern through a variety of assessment tools. Although individual trials have demonstrated a benefit, the findings are often complicated by the type of Mediterranean diet employed and the nature of the control group.21,22

\section{Effect on health}

One recent systematic review summarised the results from 58 studies (more than 1,000,000 participants) with a wide range of outcomes, including biochemical markers as well as clinical endpoints. Despite considerable heterogeneity, the authors found sufficient positive studies in the area of cardiovascular risk to conclude that there is "robust scientific evidence to support the promotion of the Mediterranean diet for both primary and secondary prevention of chronic disease and specifically of CVD".23 The review included 33 cross-sectional studies, 8 cohort studies and 16 interventional studies. No meta-analyses were performed.

Two systematic reviews with meta-analyses assessed the impact of the Mediterranean diet on CVD prevention. 5,24 The first included a meta-analysis of two randomised controlled trials (numbers of participants not given), one on secondary prevention and one on primary prevention of cardiovascular events. It found a combined risk ratio of $0.6(95 \% \mathrm{Cl} 0.5$ to 0.9$)$ in favour of the Mediterranean diet, compared with either no specific dietary advice ${ }^{25}$ or a low-fat diet. ${ }^{5}$ The researchers also conducted a meta-analysis of 12 prospective observational studies, which measured the effect of a 2-point incremental increase in adherence to the Mediterranean diet (scale 0-9). It found a risk ratio of $0.90(95 \% \mathrm{Cl} 0.86$ to 0.94$)$ for mortality from or incidence of CVD associated with each 2-point increase.

The second review looked only at primary prevention and excluded one major randomised controlled trial included in the first review, because the comparison group followed a low-fat diet, rather than having no or minimal intervention. ${ }^{24}$ The review found only one study that reported clinical endpoints, in which there was no statistically significant clinical benefit. However, it is unclear whether this study measured the effects of the Mediterranean diet, as it did not describe the intervention as such, and only included two of the seven components of the Mediterranean diet identified by the review.

Other systematic reviews have found some evidence of a protective effect against diabetes ${ }^{20}$ and metabolic syndrome. ${ }^{26}$ The former included one randomised controlled trial, nine prospective cohort studies and seven cross-sectional studies with a total of 136,846 participants. A meta-analysis showed a relative risk of developing type 2 diabetes of 0.8 ( $95 \% \mathrm{Cl} 0.7$ to 0.9 ) when comparing the lowest with highest centile of adherence. ${ }^{20}$ The review of metabolic syndrome included four randomised controlled trials (1,601 participants). ${ }^{26}$ In three trials, people assigned to the Mediterranean diet were more likely to show reversal of metabolic syndrome than people assigned to low-fat diets or usual care. In the fourth, there was no significant difference. Metabolic syndrome was assessed by different tools for different studies, with a common focus on abdominal obesity, elevated triglycerides, decreased high-density lipoprotein cholesterol, elevated blood pressure and glucose intolerance.

A study looking at epidemiological evidence for the effect of Mediterranean diet on cancer reported mixed results. Although some earlier studies suggested a reduction in colorectal cancer, the authors noted that there was a lack of definitive evidence for an association of Mediterranean diet with different types of cancer. ${ }^{27}$

A systematic review of economic analyses drew on eight studies, three of which looked at healthcare cost savings. ${ }^{28}$ One of these found that the Mediterranean diet was highly cost-effective for increasing quality adjusted life years after a first myocardial infarction. A primary prevention study found lower healthcare costs in adults following the diet, who gained an average six life-years that were lost to disability in those not following the diet. Another study in the review found a cost-effectiveness ratio of $£ 1,300$ per non-fatal myocardial infarction avoided. ${ }^{28}$ Five studies in the review looked at the costs of the diet, compared with a traditional Western diet. Their findings disagreed, with some stating that a healthy Mediterranean diet was more expensive, while others found it an 'affordable alternative', especially if it focused on grains and legumes rather than fresh fish. ${ }^{28}$ The studies did not report on adverse events.

\section{Sodium restriction}

UK guidelines advise reducing salt intake at population level to a maximum of $6 \mathrm{~g}$ per day for adults by 2015 and $3 \mathrm{~g}$ by 2025 (see Box). ${ }^{29,30}$ An estimated $65-70 \%$ of dietary sodium consumed is from manufactured foods. ${ }^{31}$ However, accurately assessing daily salt intake is difficult.

\section{Box: Sodium content}

$6 \mathrm{~g}$ sodium chloride $=2.4 \mathrm{~g}$ sodium $=260 \mathrm{mmol}$ sodium

$3 \mathrm{~g}$ sodium chloride $=1.2 \mathrm{~g}$ sodium $=130 \mathrm{mmol}$ sodium

Systematic reviews confirm that lowering sodium intake reduces blood pressure, 32,33 but direct evidence that reducing salt also reduces risk of CVD is limited.

\section{Effect on health}

One systematic review of 37 randomised controlled trials (5,508 participants) found that reducing sodium intake was associated with a reduction in systolic blood pressure of $3.4 \mathrm{mmHg}(95 \% \mathrm{Cl} 2.5$ to 4.3$) .{ }^{32}$ The same review found too few randomised controlled trials to estimate the effects of salt reduction on clinical endpoints. A meta-analysis of prospective cohort studies found that higher sodium intake was associated with increased risk of stroke (risk ratio $1.2,95 \% \mathrm{Cl} 1.08$ to 1.4 ) and fatal coronary heart disease events (risk ratio $1.3,95 \% \mathrm{Cl} 1.1$ to 1.5 ) but no significant association with all-cause mortality, CVD or coronary heart disease. A second review and meta-analysis, which included 34 randomised controlled trials (3,230 participants) of at least 4 weeks' duration of modest salt reduction in non-acutely ill adults, found slightly larger reductions in systolic blood pressure $(4.2 \mathrm{mmHg}, 95 \% \mathrm{Cl} 5.2$ to 3.2$){ }^{33}$

The reviews also looked for an effect on blood lipids, catecholamines, hormones and renal function, after earlier studies raised questions about whether reducing salt intake could be harmful. The first study found no significant adverse effect. The second found no effect on lipids, but a small physiological increase in plasma renin activity, aldosterone and noradrenaline. The authors commented that these changes were likely to attenuate over time.

A cochrane review compared the effects of a dietary sodium intake of $<120 \mathrm{mmol}$ with a sodium intake of $>150 \mathrm{mmol}$. $^{34}$ In normotensive Caucasians, lower sodium intake was associated with a decrease of $1.27 \mathrm{mmHg}(95 \% \mathrm{Cl}-1.88$ to $-0.66 ; \mathrm{p}=0.0001)$ in systolic blood pressure and $0.05 \mathrm{mmHg}$ ( $95 \% \mathrm{Cl}:-0.51$ to $0.42 ; \mathrm{p}=0.85$ ) in diastolic blood pressure (62 studies). In Caucasians with hypertension (74 studies) it led to a decrease of $5.48 \mathrm{mmHg}$ in systolic blood pressure $(95 \% \mathrm{Cl}-6.53$ to $-4.43 ; \mathrm{p}<0.00001)$ and 
$2.75 \mathrm{mmHg}$ diastolic blood pressure $(95 \% \mathrm{Cl}-3.34$ to $-2.17 ; \mathrm{p}<0.00001)$. In other ethnic population groups there were more limited data. In addition, the authors noted that there was a statistically significant increase in plasma renin, plasma aldosterone, plasma adrenaline and plasma noradrenaline, cholesterol $(0.15 \mathrm{mmol} / \mathrm{L})$ and triglycerides $(0.08 \mathrm{mmol} / \mathrm{L})$ reported. However, the studies were not designed to measure long-term health outcomes. ${ }^{34}$

A clinical review cast doubt on the recommendations for "a generalised and indiscriminate reduction of salt intake at population level".4 The authors question the association between incidence of hypertension and measurement of salt intake by urine analysis. They raise the possibility that reduced salt intake may increase mortality. A separate article concluded that unexpected findings of a relationship between lower salt and higher mortality could be a result of reverse causality in people already ill with diabetes or heart failure. ${ }^{2}$ The author concluded that calls to abandon salt guidelines were "based on flawed analyses of data from observational data studies that were not planned to study sodium relationships".

While more long-term randomised controlled trials with clinical outcomes are desirable, the practicalities may be prohibitive. One group of researchers estimate that an appropriate trial would need to randomise around 28,000 people, with a follow-up of at least 5 years. ${ }^{2}$

\section{Effectiveness of dietary advice}

The authors of a systematic review of randomised controlled trials of any type of dietary advice conclude that advice "appears to be effective in bringing about modest beneficial changes". ${ }^{35}$ The review included 44 trials (18,175 participants) with a median follow-up period of 12 months. More than half (29/44) of the trials were conducted in the USA. The advice centred on reducing salt and fat intake and increasing fruit and vegetable intake. It was delivered in a variety of ways, including individual and group sessions, and using written materials. In a meta-analysis, compared with no advice, dietary advice increased fruit and vegetable intake by 1.18 servings a day (baseline not given, $95 \% \mathrm{Cl} 0.7$ to 1.7). Advice reduced cholesterol levels by a small amount (total cholesterol reduced by $0.15 \mathrm{mmol} / \mathrm{L}[95 \% \mathrm{Cl}$ 0.1 to 0.2$]$, and LDL cholesterol by $0.16 \mathrm{mmol} / \mathrm{L}[95 \% \mathrm{Cl} 0.1$ to 0.2$]$ from an initial total cholesterol of 4.4 to $7.4 \mathrm{mmol} / \mathrm{L}$ ), systolic blood pressure by $2.61 \mathrm{mmHg}(95 \% \mathrm{Cl} 1.3$ to 3.9$)$ and diastolic blood pressure by $1.45 \mathrm{mmHg}$ ( $95 \%$ Cl 0.7 to 2.2 ) from an initial range of $125 / 84 \mathrm{mmHg}$ to $161 / 98 \mathrm{mmHg}$.

\section{Conclusion}

It is not surprising that the evidence base for dietary interventions is largely observational. Randomised controlled trials powered to show a difference in clinical outcomes in the general population would need to be large and last for many years; hence, the randomised controlled trial evidence for clinically-important effects of fruit and vegetables, Mediterranean diet and reduced salt is limited. However, the absence of such trial evidence does not mean that interventions are ineffective.

Epidemiological evidence suggests that increased fruit and vegetable intake may have a small effect on mortality and cardiovascular disease (CVD). Although reducing salt intake may lower blood pressure, the absolute benefits are largely based on extrapolation of the impact of lowering blood pressure rather than a reduction in mortality or morbidity. The benefit of reducing salt consumption appears to be greater for people who have raised blood pressure. However, the impact of low sodium intake on other biochemical markers has led to some debate about recommendations for a very low level of sodium consumption. The Mediterranean diet is supported by evidence from clinical trials and observational data and has been shown to lower the risk of CVD and diabetes. However, difficulties over the definition of the components of such a diet make it challenging to translate the findings into practical advice. Nevertheless, based on the evidence it would seem sensible to aim for a balanced diet with plenty of fruit and vegetables, beans, whole grains, olive oil, fish and a limit on the amount of salt.

The question remains as to which are the most effective strategies for bringing about dietary changes. Individual dietary advice has a limited effect and tends not to be cost-effective. Reduced sodium consumption is likely to be achievable only through meaningful measures to reduce the amount of salt in processed food.

1. National Institute for Health and Care Excellence, 2014. Lifestyle advice on diet and physical activity [online]. Available: http://pathways.nice.org.uk/pathways/ diet/lifestyle-advice-on-diet-and-physical-activity\#content=viewnode\%3Anodes-diet-advice-for-adults [Accessed 17 December 2014]

2. Whelton PK et al. Sodium, blood pressure, and cardiovascular disease: furthe evidence supporting the American Heart Association sodium reduction recommendations. Circulation 2012; 126: 2880-9.

M 3. Wang X0. Fruit and vegetable consumption and mortality from all causes, cardiovascular disease, and cancer: systematic review and dose-response meta-analysis of prospective cohort studies. BMJ 2014; 349: 94490.

4. Stolarz-Skrzypek $\mathrm{K}$ et al. Blood pressure, cardiovascular outcomes and sodium intake, a critical review of the evidence. Acta Clin Belg 2012; 67: 403-10.

M 5. Martínez-González MA, Bes-Rastrollo M. Dietary patterns, Mediterranean diet, and cardiovascular disease. Curr Opin Lipidol 2014; 25: 20-6.

6. Public Health England, 2014. National diet and nutrition survey: results from years 1-4 (combined) of the rolling programme (2008/2009-2011/12) [online]. Available: https://www.gov.uk/government/uploads/system/uploads/ attachment_data/file/310997/NDNS_Y1_to_4_UK_report_Executive_summary. pdf [Accessed 17 December 2014]

7. European Society of Cardiology, 2012. European guidelines on CVD prevention in clinical practice [online]. Available: http://www.escardio.org/GUIDELINESSURVEYS/ESC-GUIDELINES/Pages/cvd-prevention.aspx [Accessed 17 December 2014].

8. Health Canada, 2011. Eating well with Canada's food guide [online]. Available: http://www.hc-sc.gc.ca/fn-an/alt_formats/hpfb-dgpsa/pdf/ food-guide-aliment/view_eatwell_vue_bienmang-eng.pdf [Accessed 17 December 2014].

9. US Department of Agriculture, US Department of Health and Human Services, 2010. Dietary guidelines for Americans [online]. Available: http://www.cnpp.
susda.gov/sites/default/files/dietary_guidelines_for_americans/PolicyDoc.pd [Accessed 17 December 2014].

10. Australian Government, National Health and Medical Research Council, Department of Health and Ageing, 2013. Eat for health: Australian dietary guidelines [online]. Available: http://www.nhmrc.gov.au/_files_nhmrc/ publications/attachments/n55a australian dietary guidelines summary_131014.pdf [Accessed 17 December 2014].

11. Doctors of $B C$, Ministry of Health (British Columbia), Guidelines and Protocols Advisory Committee, 2014. Cardiovascular disease-primary prevention [online]. Available: http://www.bcguidelines.ca/pdf/cvd.pdf [Accessed 17 December 2014]

M 12. Hartley $L$ et al. Increased consumption of fruit and vegetables for the primary prevention of cardiovascular diseases. Cochrane Database Syst Rev 2013; 6 CD009874. D0I:10.1002/14651858.CD009874.pub2 [Last assessed as up-to-date 27 September 2012].

M 13. Hu D et al. Fruits and vegetables consumption and risk of stroke: a metaanalysis of prospective cohort studies. Stroke 2014; 45: 1613-9.

14. Martínez-González MA et al. Low consumption of fruit and vegetables and risk of chronic disease: a review of the epidemiological evidence and temporal trends among Spanish graduates. Public Health Nutr 2011; 14: 2309-15.

M 15. Norat T et al. Fruits and vegetables: updating the epidemiological evidence for the WCRF/AICR lifestyle recommendations for cancer prevention. Cancer Treat Res 2014; 159: 35-50.

M 16. Cooper AJ et al. Fruit and vegetable intake and type 2 diabetes: EPICInterAct prospective study and meta-analysis. Eur J Clin Nutr 2012; 66: 1082-92.

$M$ 17. Carter $P$ et al. Fruit and vegetable intake and incidence of type 2 diabetes mellitus: systematic review and meta-analysis. BMJ 2010; 341: c4229.

18. Cobiac LJ et al. Cost-effectiveness of interventions to promote fruit and vegetable consumption. PLOS ONE 2010; 5: e14148. 
19. Gulliford MC et al. Cost-effectiveness of a universal strategy of brief dietary intervention for primary prevention in primary care: population-based cohort study and Markov model. Cost Eff Resour Alloc 2014; 12: 4.

M 20. Koloverou E et al. The effect of Mediterranean diet on the development of type 2 diabetes mellitus: a meta-analysis of 10 prospective studies and 136,846 participants. Metabolism 2014; 63: 903-11.

$R$ 21. Estruch $R$ et al. Primary prevention of cardiovascular disease with a Mediterranean diet. N EnglJ Med 2013; 368: 1279-90.

22. Appel LJ, Van Horn L. Did the PREDIMED trial test a Mediterranean diet? N Engl Med 2013; 368: 1353-4.

23. Grosso $\mathrm{G}$ et al. Mediterranean diet and cardiovascular risk factors: a systematic review. Crit Rev Food Sci Nutr 2014; 54: 593-610.

M 24. Rees $\mathrm{K}$ et al. 'Mediterranean' dietary pattern for the primary prevention of cardiovascular disease. Cochrane Database Syst Rev 2013; 8: CD009825. DOI:10.1002/14651858.CD009825.pub2 [Last assessed as up-to-date 15 October 2012].

25. Kris-Etherton P et al. AHA Science Advisory: Lyon Diet Heart Study. Benefits of a Mediterranean-style, National Cholesterol Education Program/American Heart Association Step I Dietary Pattern on cardiovascular disease. Circulation 2001; 103: $1823-5$

26. Esposito $\mathrm{K}$ et al. Mediterranean diet and metabolic syndrome: an updated systematic review. Rev Endocr Metab Disord 2013; 14: 255-63.

27. Kontou $\mathrm{N}$ et al. The Mediterranean diet in cancer prevention: a review. J Med Food 2011; 14: 1065-78.

28. Saulle R et al. Cost and cost-effectiveness of the Mediterranean diet: results of a systematic review. Nutrients 2013; 5: 4566-86.
29. National Institute for Health and Care Excellence, 2010. Prevention of cardiovascular disease (PH25) [online]. Available: http://www.nice.org.uk/ guidance/ph25 [Accessed 17 December 2014].

30. Scottish Intercollegiate Guidelines Network, 2007. Risk estimation and the prevention of cardiovascular disease [online]. Available: http://www.sign.ac.uk/ pdf/sign97.pdf [Accessed 17 December 2014].

31. Scientific Advisory Committee on Nutrition, 2003. Salt and health [online] Available: https://www.gov.uk/government/uploads/system/uploads/ attachment_data/file/338782/SACN_Salt_and_Health_report.pdf [Accessed 17 December 2014].

M 32. Aburto $\mathrm{NJ}$ et al. Effect of lower sodium intake on health: systematic review and meta-analyses. BMJ 2013; 346: f1326.

M 33. He Fj et al. Effect of longer-term modest salt reduction on blood pressure. Cochrane Database Syst Rev 2013; 4: CD004927. D0l:10.1002/14651858. CD004937.pub2 [Last assessed as up-to-date 28 February 2013].

M 34. Graudal NA et al. Effects of low sodium diet versus high sodium diet on blood pressure, renin, aldosterone, catecholamines, cholesterol, and triglyceride. Cochrane Database Syst Rev 2011; 11: CD004022. D0I:10.1002/14651858. CD004022.pub3. [Last assessed as up-to-date 4 October 2011].

M 35. Rees K et al. Dietary advice for reducing cardiovascular risk. Cochrane Database Syst Rev 2013; 12: CD002128. D0I:10.1002/14651858.CD002128.pub5 [Last assessed as up-to-date 27 April 2011]

DOI: $10.1136 / d t b .2015 .1 .0301$ 